\title{
Single-Layer WEBs: Intrasaccular Flow Disrupters for Aneurysm Treatment-Feasibility Results from a European Study
}

\author{
(D). Caroff, C. Mihalea, J. Klisch, C. Strasilla, A. Berlis, T. Patankar, W. Weber, D. Behme, E.A. Jacobsen, T. Liebig, S. Prothmann,
} C. Cognard, T. Finkenzeller, J. Moret, and L. Spelle

\begin{abstract}
BACKGROUND AND PURPOSE: The safety and efficiency of the dual-layer Woven EndoBridge (WEB) device has already been published. However, this international multicenter study sought to evaluate the safety of single-layer devices, which are the newest generation of the WEB intrasaccular flow-disrupter family. They have been designed to offer smaller-sized devices with a lower profile to optimize navigability and delivery, which may, in turn, broaden their range of use.
\end{abstract}

MATERIALS AND METHODS: Data from all consecutive patients treated with a single-layer WEB device, in 10 European centers from June 2013 to May 2014 were included. Clinical presentations, technical details, intra- and perioperative complications, and outcomes at discharge were recorded. Clinical and angiographic data at last follow-up were also analyzed when available.

RESULTS: Ninety patients with 98 WEB-treated aneurysms were included in this study. In 93 cases (95\%), WEB placement was possible. Complete occlusion at the end of the procedure was obtained in 26 instances (26\%). Additional treatment during the procedure (coiling and/or stent placement) was necessary in 12 cases (12.7\%). Procedure-related complications occurred in 13 cases, leading to permanent neurologic deficits in 4 patients (4.4\%). Early vascular imaging follow-up data were available for 44 patients (57\%), with an average time interval of 3.3 months. Treatment-related morbidity and mortality rates at last follow-up were $2.2 \%$ and $1.1 \%$, respectively.

CONCLUSIONS: In this study, the feasibility and safety of the single-layer WEB device was comparable with that of the double-layer. However, further studies are needed to evaluate long-term efficacies.

ABBREVIATIONS: $\mathrm{DL}=$ dual-layer; $\mathrm{WEB}=$ Woven EndoBridge; $\mathrm{SL}=$ single-layer; SLS = single-layer sphere.

$\mathbf{T}$ he Woven EndoBridge dual-layer device (WEB-DL; Sequent Medical, Aliso Viejo, California) is an intrasaccular flowdisruption device, initially developed to treat wide-neck bifurcation intracranial aneurysms. It has proved to be a highly feasible,

Received January 6, 2015; accepted after revision February 25.

From the Interventional Neuroradiology NEURI Center (J.C., C.M., J.M., L.S.), Beaujon Hospital, Clichy, France; Department of Neurosurgery (C.M.), University of Medicine and Pharmacy "Victor Babes," Timisoara, Romania; Department of Neuroradiology (J.K., C.S.), Helios General Hospital, Erfurt, Germany; Department of Diagnostic and Interventional Neuroradiology (A.B.), Klinikum Augsburg, Augsburg, Germany; MRI Department (T.P.), Leeds Teaching Hospitals National Health Service Trust, Leeds, UK; Interventional Neuroradiology (W.W.), Ruhr University Medical Center, Bochum, Germany; Departments of Radiology and Neuroradiology (D.B.), Klinikum Vest, Recklinghausen, Germany; Interventional Neuroradiology (E.A.J.), Oslo University Hospital, Oslo, Norway; Interventional Neuroradiology (T.L.), Universitatsklinik Koeln, Köln, Germany; Abteilung für Diagnostische und Interventionelle Neuroradiologie (S.P.), Klinikum rechts der Isar, Technische Universität München, München, Germany; Interventional Neuroradiology (C.C.), Centre Hospitalier Universitaire Hôpital Purpan, Toulouse, France; and Interventional Neuroradiology (T.F.), Klinikum Nürnberg Süd, Nürnberg, Germany.

Please address correspondence to Jildaz Caroff, MD, Hôpital Beaujon, 100, Boulevard Général Leclerc, 92110 Clichy, France; e-mail: Jildaz.caroff@bjn.aphp.fr; @jildazz

- Indicates open access to non-subscribers at www.ajnr.org

http://dx.doi.org/10.3174/ajnr.A4369

safe, ${ }^{1-4}$ and efficient ${ }^{5}$ technique, particularly dedicated to the treatment of aneurysms challenging to treat with standard coiling.

The single-layer WEB (WEB-SL) device is the newest generation of the WEB family. Improved microbraiding technology allows a reduction in device size and profile, which optimizes navigability (through smaller catheters) and delivery. The purpose of this study was to evaluate the feasibility and safety of this new device, which has been available in Europe since 2013.

\section{MATERIALS AND METHODS Population}

All patients with planned treatment with the WEB-SL device, in the 10 participating centers from June 2013 to May 2014, were consecutively included in this study. No exclusion criteria were used. The decision to use the WEB-single-layer device was made locally by the interventional teams and was based on aneurysm characteristics (size, wide-neck, bifurcation location, and so forth) in each case. It mostly corresponded to bifurcation aneurysms with aspect ratios of $<1.2$, in which adjunctive techniques to coiling would have been required. ${ }^{6}$ Informed consent was obtained from all patients. 
A
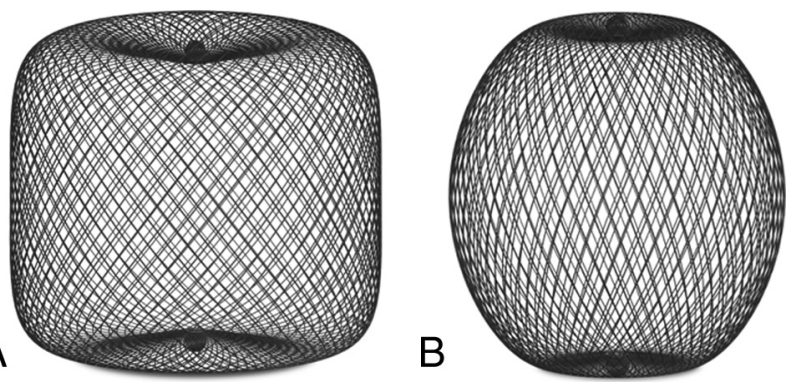

FIG 1. WEB-SL device family. $A$, The barrel-shaped WEB-SL, similar to the WEB-DL. $B$, The spheric WEB-SLS.

\section{WEB Devices}

The WEB-SL and WEB-single-layer sphere (SLS) are the latest generation of WEB devices and became available for use in 2013 (Fig 1). The WEB-SL is barrel-shaped (similar to the WEB-DL) and the WEB-SLS is spheric. They are available in various diameters, from 4 to $11 \mathrm{~mm}$ and heights from 3 to $9 \mathrm{~mm}$. Unlike the WEB-DL, the WEB-SL and SLS devices can contain from 144 wires in the 4-mmdiameter devices to 216 wires in the $11-\mathrm{mm}$-diameter devices. They present a lower profile and were designed for use with the VIA-27 and VIA-33 catheters (Sequent Medical), which are 3F and 3.4F (outer diameter), respectively. The VIA-33 is dedicated to the WEB-SL and SLS devices with diameters of $10 \mathrm{~mm}$ and above.

\section{Treatment Technique}

Endovascular treatment was performed with the patient under general anesthesia and systemic heparinization. By the end of the procedure, the heparin therapy was discontinued but not reversed. Antiplatelet medications were used pre- and postoperatively, depending on local protocols and angiographic results. In $94 \%$ of cases, VIA catheters were used for WEB delivery. The use of antiplatelet agents in the pre- and postoperative periods was decided locally and for each case by the operator. Even if the use of an antiplatelet agent is not mandatory with the WEB device, depending on local habits, some patients were premedicated with aspirin and clopidogrel in case of a strategy shift to stent-assisted coiling.

\section{Data Collection}

For each aneurysm, we collected the following data retrospectively: age, sex, aneurysm location and biometry (maximum diameter, neck size, aspect ratio), rupture status, the modified Rankin Scale score at the time of admission and discharge, modalities of treatment (WEB type and size, associated medication and treatment), intraoperative complications (aneurysm rupture, thromboembolic events, deployment failure, WEB protrusion or migration), and postoperative complications (delayed bleeding, thromboembolic events). Immediate postoperative DSA, 3D rotational angiography, and VasoCT (Philips Healthcare, Best, the Netherlands) ${ }^{7}$ images were collected, along with any available follow-up imaging data (DSA, CTA, MRA).

\section{Data Analysis}

Data were self-reported by the different centers, but the principal investigators (J.C. and L.S.) performed a blinded review of the clinical and angiographic data, as an independent core laboratory not involved in patient care. Adverse events were adjudicated in-

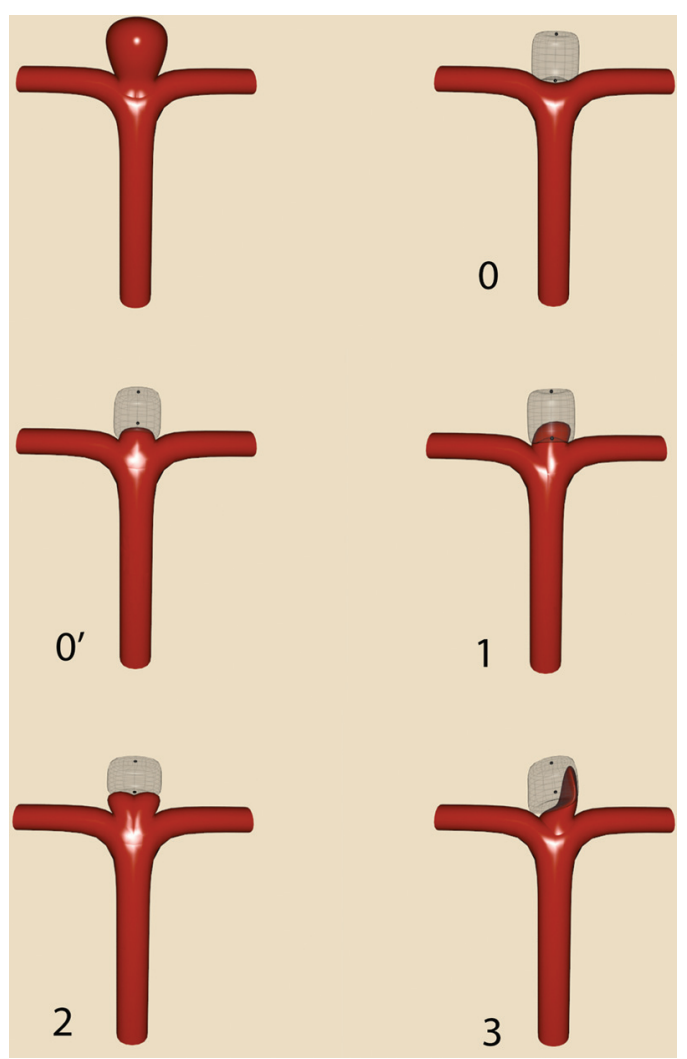

FIG 2. WEB occlusion scale. Grade 0 indicates complete occlusion. Grade $0^{\prime}$ is similar but with opacification of the proximal recess, which may also be considered complete occlusion. Grade 1 signifies opacification inside the WEB. Grade 2 denotes a neck remnant. Grade 3 indicates an aneurysm remnant with contrast agent inside the sac between the wall and the WEB device.

dependently from operators. Permanent morbidity and mortality rates, subsequent to treatment, were evaluated at discharge and at follow-up when possible. Morbidity was defined as an mRS of $>1$. When the preoperative mRS was $>1$, morbidity was defined by any increase in the mRS score.

The principal investigators also independently assessed the degree of aneurysmal occlusion, by using a modified version of the Montreal 5-grade scale as previously published. ${ }^{8}$ The Beaujon Occlusion Scale Score is described as follows: $0=$ no residual flow inside the aneurysm or the WEB, $0^{\prime}=$ opacification of the proximal recess of the WEB, 1 = residual flow inside the WEB, $2=$ neck remnant, $3=$ aneurysm remnant, and $1+3=$ contrast media depicted inside and around the device (Fig 2).

\section{RESULTS \\ Population}

Ninety patients ( 60 women and 30 men; age range, $26-83$ years; median age, 55 years) with 98 WEB-treated aneurysms were included in this study. Of these, 65 aneurysms were unruptured (66\%) (Fig 3). They were treated in 10 European centers with a median of 7.5 WEB implants per center.

It was determined that 38 aneurysms were located along the middle cerebral artery $(38.8 \%) ; 21$, along the anterior communicating artery $(21.4 \%) ; 19$, along the basilar artery (19.4\%); and 15, along the supraclinoid internal carotid artery (15.3\%). Aneurysm size varied from 3.7 to $39 \mathrm{~mm}$ (mean, $8.9 \pm 5.5 \mathrm{~mm}$ ), neck size 

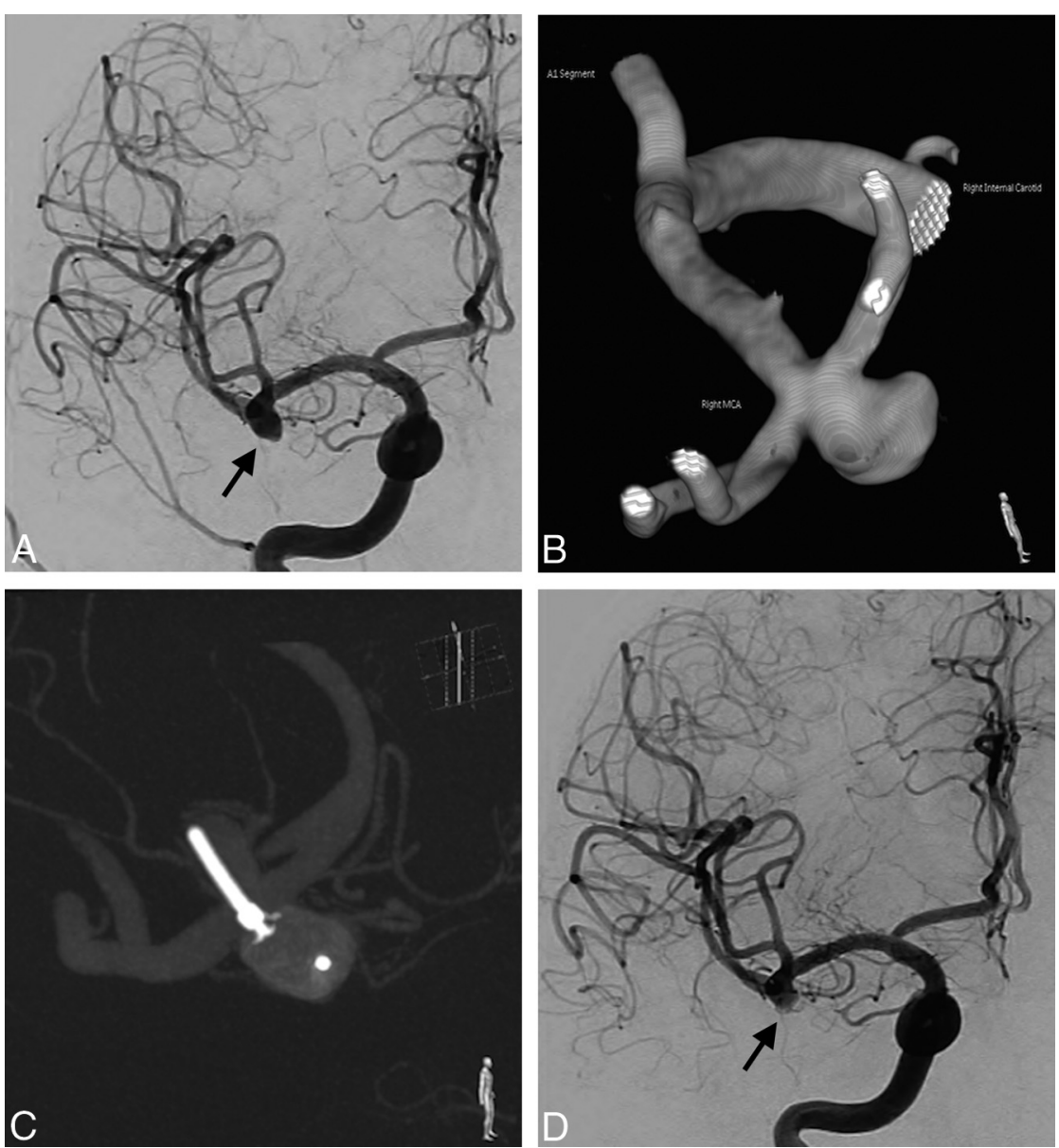

FIG 3. A 65-year-old man was admitted for the treatment of an asymptomatic aneurysm. Preoperative DSA showed a 6-mm right MCA bifurcation aneurysm (A). B, 3D rotational angiography before treatment. $C$, VasoCT before detachment confirmed the correct positioning of the WEBSL. D, After WEB-SL deployment, postoperative DSA showed partial occlusion and stagnation of contrast agent inside the aneurysm. The Beaujon Occlusion Scale Score is 3.

varied from 2.0 to $12.3 \mathrm{~mm}$ (mean, $5.2 \pm 2.1 \mathrm{~mm}$ ), and the aspect ratio, defined as depth/neck, varied from 0.6 to $7.6 \mathrm{~mm}$ (mean, $1.6 \pm 1.0 \mathrm{~mm})$.

\section{Treatment Feasibility}

The WEB-SL device was successfully deployed inside the aneurysmal sac in 93 cases (95\%). Deployment failures were attributed to device migration during detachment in 1 instance, while there were difficulties in achieving satisfactory positioning of the WEB before detachment in the other 4 cases. Of those 5 aneurysms, 3 were successfully treated with stent-assisted coiling, 1 case required a WEB-DL, and simple coiling was used in 1 case.

\section{Additional Treatment}

The necessity of additional coiling had been anticipated preoperatively, to manage large or giant aneurysms in 4 patients and to ensure immediate occlusion of a daughter bleb, in a fifth patient with a ruptured aneurysm. In 7 cases (8.2\%), unplanned additional treatment had been required. In 5 of these cases, stent deployment was needed because of WEB protrusion into the parent artery (Fig 4). In the remaining 2 cases, additional coiling was performed to complete aneurysm occlusion after WEB deployment because of the presence of aneurysm remnants.

\section{Protrusion}

Postdelivery, major protrusion occurred in 14 cases. It required balloon remodeling in 5 and additional stent placement in 5 . In 3 other cases, it led to clot formation, successfully treated with abciximab infusion in 2 cases and with mechanical thrombectomy in 1 . In the last case, 1 branch occlusion occurred, resulting in an ischemic infarction. In 29 cases, a protrusion of only the proximal marker was depicted at the level of the bifurcation. In those situations, a postoperative antiplatelet regimen was prescribed at the physician's discretion.

\section{Platelet Antiaggregation Therapy}

One or 2 platelet antiaggregation agents were administered preoperatively to 27 patients $(30 \%)$, while 60 patients $(67 \%)$ were still given at least 1 antiplatelet therapy at discharge, mostly because of slight device protrusion in the parent artery.

\section{Complication Rate}

Procedure-related complications occurred in 13 cases. We observed 6 intraoperative thromboembolic complications, 4 of which were successfully managed ( 2 treated by intra-arterial infusion of abciximab; 1, by stent deployment; and another, by performing a thrombectomy by using a Solitaire stent [Covidien, Irvine, California]). The other 2 patients had arterial occlusion and hemiplegia. One recovered partially and was mRS 1 at last follow-up, and the other was rated mRS 3 .

We also observed 5 hemorrhagic complications. In 1 case, an aneurysmal rupture occurred due to a wire perforation but was immediately stopped after WEB delivery, and the patient remained asymptomatic. In another case, an occipital lobe parenchymal bleed occurred 4 days after the treatment of an anterior communicating artery aneurysm, and the patient still had hemianopsia at last followup. The patient had a history of uncontrolled hypertension and received double-antiplatelet therapy as a premedication.

The other 3 were ruptured cases, and early follow-up showed new parenchymal or subarachnoidal bleeding, which led to severe disability in 1 patient, seizures in the second patient, and death in the last patient. In the first one, an aneurysmal bleb was occluded by 1 coil, then the aneurysm itself was treated with a WEB, stagnation was obtained inside the sac, but 6 hours later a large parenchymal hematoma was depicted. In the second one, with a multilobulated anterior communicating artery aneurysm, only partial occlusion was obtained after WEB delivery. A nonoccluded part of the aneurysm, initially misjudged as a nonpotential source of bleeding, was secondarily coiled after rebleeding was revealed by seizures. The last case was one of major and diffuse SAH. Three potential ruptured aneurysms were discovered; 1 was treated with coils, and other 2, with the WEB-SL. 

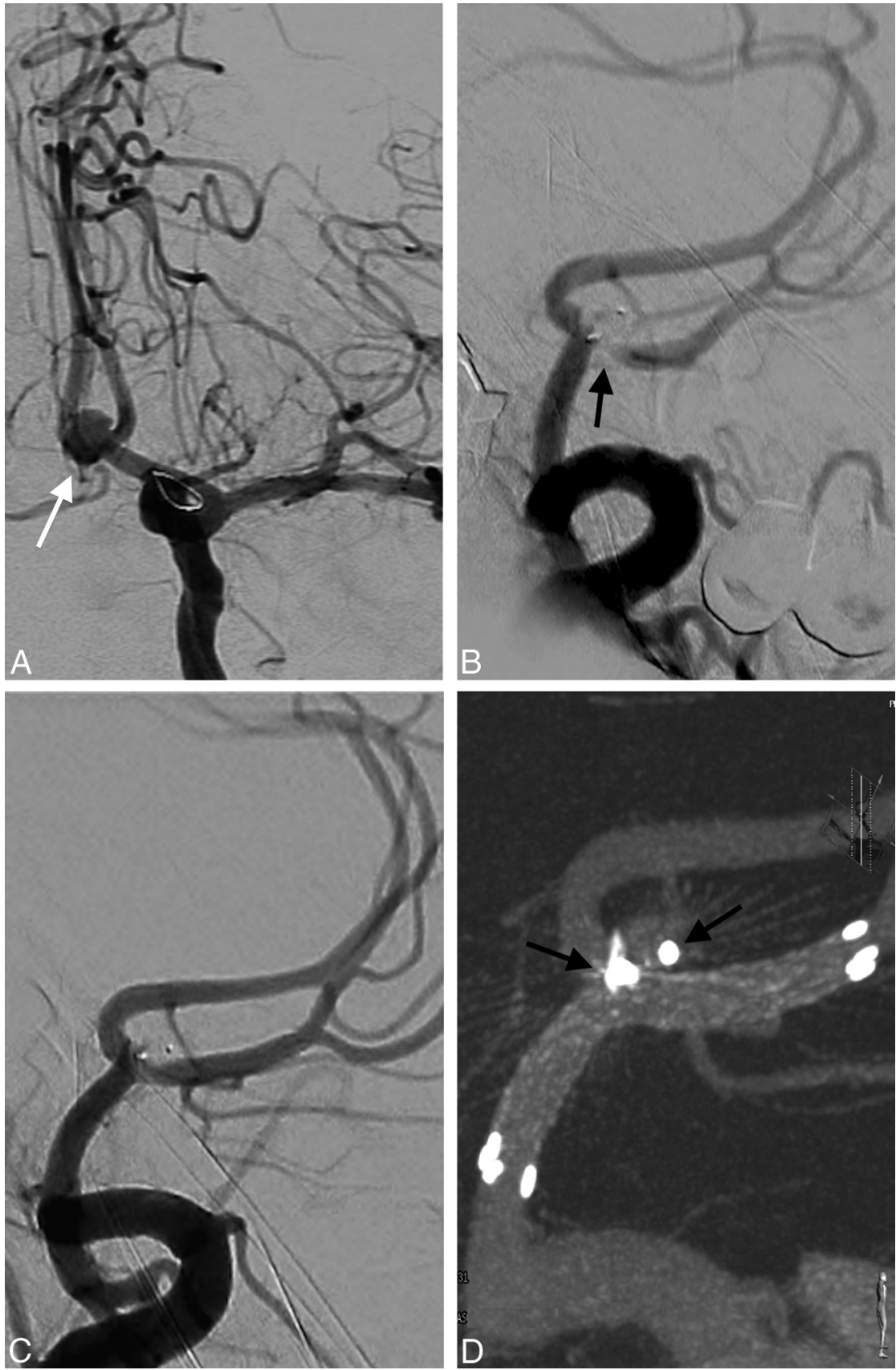

FIG 4. A 59-year-old women was admitted for the treatment of an unruptured anterior communicating artery aneurysm. A, Preoperative DSA. B, After WEB-SL deployment, a protrusion occurred in the right $\mathrm{A} 2$ segment, leading to a significant stenosis (black arrow). The proximal marker is also depicted within the parent artery. C, A laser cut Enterprise stent (Codman \& Shurtleff, Raynham, Massachusetts) was then delivered to restore the caliber of the artery. The Beaujon Occlusion Scale Score is 0 . D, Final VasoCT confirmed satisfactory positioning of the stent without any protrusion of the WEB (arrows show the 2 radio-opaque markers of the WEB-SL).

Massive rebleeding predominantly located in the right Sylvian fissure occurred 3 hours after treatment, leading to death. The lesions that were most likely to be the cause of the recurrent fatal hemorrhage were probably 1 of the 2 right MCA aneurysms. One was treated with coils, and 1, with the WEB-SL. After treatment, those 3 patients were kept under at least 1 antiplatelet agent because of device protrusion.

A distal migration of the WEB device during removal, due to an incomplete detachment, was noted in 1 instance. It was impossible to recapture the device in the M1 segment. While we tried to recapture it, it finally migrated into an M2 branch in a nondeployed fashion without flow repercussions. The patient remained asymptomatic under double antiplatelet therapy, and no ischemic lesions were observed on imaging follow-up.

One patient was mRS 1 at discharge due to a postoperative iliac artery occlusion.

Data from early clinical follow-ups were available for 52 patients (58\%), with an average time interval of 3.8 months. Treatment-related morbidity and mortality rates at last follow-up were $2.2 \%$ and $1.1 \%$, respectively.

\section{Immediate Results}

Immediate angiographic evaluation showed 27 cases (28\%) of complete occlusion, 2 neck remnants, and a stagnation of iodine contrast agent in the aneurysm in all other cases.

\section{Angiographic Follow-Up}

Short-term vascular imaging follow-ups were available in 69 cases (70\%), including DSA data in 54 cases, and the average time interval to follow-up was 3.3 months. Satisfactory results (complete occlusion or small neck remnants) were achieved in 45 patients (65\%). In 8 cases, contrast opacification agent was still observed inside the device at that time.

\section{DISCUSSION}

The work presented here is the largest WEB study undertaken to date, wherein WEB-SL devices were used to treat aneurysms that would normally be considered difficult to treat with traditional endovascular approaches. Most of the treated aneurysms were wide-neck (average, $5.1 \mathrm{~mm}$ ), with unfavorable anatomy (average aspect ratio, 1.6). Brinjikji et $\mathrm{al}^{6}$ defined 2 thresholds based on the aspect ratio value, to define the possible strategy of endovascular treatments. They reported that in cases with a value below 1.2 (39\% of cases in our study), adjunctive techniques are almost always necessary. Usually, an aspect ratio of $>1.6$ implied that adjunctive techniques were not required. In our study, $69 \%$ of cases presented a lower ratio than this.

Most lesions were bifurcation aneurysms (86\%). Approximately $40 \%$ were middle cerebral artery aneurysms, $20 \%$ were anterior communicating artery aneurysms, and 20\% were basilar tip aneurysms.

Within this study, $34 \%$ of cases were ruptured aneurysms, which is much higher than the percentage in other WEB series. In 
contrast to our previous observations, from investigation of a smaller cohort, ${ }^{8} 3$ patients had rebleeding after WEB treatment. At the end of the procedure, contrast agent stagnation was detected in the proximal part of those aneurysms, but complete occlusion was not yet achieved. After the endovascular treatment, those 3 patients were kept under at least 1 antiplatelet agent, and this could have influenced this relatively high rebleeding rate.

Despite the fact that aneurysm geometry was unfavorable in our study, the procedure-related complication rate was low (13\%) and comparable with that obtained from the largest WEB-DL study $(10.8 \%){ }^{1}$ The rupture rate during treatment was low (1\%) and was, in fact, unrelated to WEB delivery but was caused by guidewire perforation during catheterization of the sac. Bleeding was controlled by rapid WEB delivery, and the patient remained asymptomatic.

Treatment-related morbidity and mortality rates at last follow-up were $2.2 \%$ and $1.1 \%$, respectively. Finally, safety results from this WEB-SL study are similar to those in other published WEB-DL studies. Indeed those authors reported morbidity ( $\mathrm{mRS}$ of $>2$ ) rates between $1.3 \%$ and $6.7 \%$ and mortality rates between $0 \%$ and $2.2 \%$. ${ }^{1,3,5}$ These results are also comparable with those obtained from neurosurgical clipping of unruptured aneurysms (6.7\% overall morbidity, $1.7 \%$ mortality $)^{9}$ and non-WEB endovascular treatment of unruptured aneurysms (2.5\% morbidity, $1.8 \%$ mortality). ${ }^{9}$

An antiplatelet premedication was used in $30 \%$ of cases (aspirin and clopidogrel) because if during a procedure, WEB treatment appears to be unsuitable, another alternative therapy can be stent-assisted coiling for these wide-neck aneurysms. In $67 \%$ of cases, at least 1 antiplatelet agent was still used at discharge. It was given either in cases of device protrusion in the parent artery or sometimes only because of proximal marker protrusion, though the need for antiplatelets in those situations is not mandatory.

This study was not intended for the evaluation of anatomic results subsequent to WEB-SL treatment. Early follow-up vascular imaging was available in a large number of cases (70\%), and it consisted of DSA data in half of these cases. These data demonstrated a low rate of satisfactory results (65\%), which could probably improve during follow-up because in some cases, stagnation was depicted inside the WEB and patients were still kept under antiplatelet therapy at that time. In a WEB-DL $\operatorname{article}^{5}$ with a short-term imaging follow-up (average length, 5.0 months), authors reported complete occlusion in $57 \%$ of cases; in our series, it was obtained in only $38 \%$ of cases. However, as with flow-diverter stents, delayed thrombosis should be expected. Therefore, results must be reviewed after longer time intervals.

This study has several limitations. First the number of treated cases for each center is low and mostly corresponds to an initial experience for the operators with this new device. The complications were self-reported. Angiographic outcome is now still limited, but it appeared important for us to report these preliminary results before analysis of long-term follow-up imaging to evaluate the efficiency of the WEB-SL device.

\section{CONCLUSIONS}

In this study, the feasibility and safety of the single-layer WEB device was comparable with that of the double-layer device. However, further studies are needed to evaluate long-term efficacies.

\section{ACKNOWLEDGMENTS}

The authors thank Dr Francesco D'Argento from Policlinico A. Gemelli in Roma for his elegant work on the illustrations.

Disclosures: Joachim Klisch—RELATED: Consulting Fee or Honorarium: consultant to Sequent Medical.* Ansgar Berlis—RELATED: Consulting Fee or Honorarium: consultancy agreement for proctoring with Sequent Medical; UNRELATED: Consultancy: consultancy agreements for proctoring with Covidien and MicroVention; Payment for Lectures (including service on Speakers Bureaus): honorarium for lectures from Penumbra and Stryker. Tufail Patankar-UNRELATED: Travel/Accommodations/ Meeting Expenses Unrelated to Activities Listed: travel expense paid by Sequent Medical to present Web data in Val d'Isère 2015; Other: proctor for Sequent Medical. Werner Weber-UNRELATED: Payment for Lectures (including service on Speakers Bureaus): Sequent Medical (lectures); Other: Sequent Medical (proctoring). Daniel Behme-RELATED: Consulting Fee or Honorarium: speaking honoraria, German Society of Neurosurgery meeting in Dresden 2014; Support for Travel to Meetings for the Study or Other Purposes: Sequent Medical; UNRELATED: Payment for Manuscript Preparation: Penumbra, Comments: Separator 3D Series; Travel/Accommodations/Meeting Expenses Unrelated to Activities Listed: Penumbra, Comments: Anatomy Biology Clinical correlations - Working group in Interventional Neuroradiology (ABC WIN) meeting in Val d'Isère 2014 and 2015. Eva Astrid Jacobsen-RELATED: Support for Travel to Meetings for the Study or Other Purposes: MicroVention, Comments: registration fee to Live Interventional Neuroradiology and Neurosurgery Course (LINNC) meeting in Paris 2014. Thomas Liebig—RELATED: Consulting Fee or Honorarium: proctoring and consulting for Sequent Medical; Support for Travel to Meetings for the Study or Other Purposes: Sequent Medical, Comments: reimbursement of traveling and meeting admission fees; UNRELATED: Consultancy: consulting for Acandis, MicroVention, and Stryker. Sascha Prothmann—UNRELATED: Consultancy: phenox, Bochum, Germany (proctor); Payment for Lectures (including service on Speakers Bureaus): Boston Scientific, Covidien, Comments: oral presentation (workshop) at Third Munich Aortic and Carotid conference 2013 (Boston Scientific), oral presentation (workshop) at European Society of Neuroradiology Annual Meeting, Frankfurt, 2013 (Covidien). Christophe Cognard-UNRELATED: Consultancy: Stryker, MicroVention, Codman, Covidien. Jacques Moret-UNRELATED: Board Membership: Covidien; Consultancy: Covidien, MicroVention. Laurent Spelle-UNRELATED: Consultancy: Stryker, Sequent Medical, Medtronic. *Money paid to the institution.

\section{REFERENCES}

1. Papagiannaki C, Spelle L, Januel AC, et al. WEB intrasaccular flow disruptor-prospective, multicenter experience in 83 patients with 85 aneurysms. AJNR Am J Neuroradiol 2014;35:2106-11

2. Pierot L, Liebig $\mathrm{T}$, Sychra V, et al. Intrasaccular flow-disruption treatment of intracranial aneurysms: preliminary results of a multicenter clinical study. AJNR Am J Neuroradiol 2012;33:1232-38

3. Pierot L, Klisch J, Cognard C, et al. Endovascular WEB flow disruption in middle cerebral artery aneurysms: preliminary feasibility, clinical, and anatomical results in a multicenter study. Neurosurgery 2013;73:27-34; discussion 34-35

4. Lubicz B, Mine B, Collignon L, et al. WEB device for endovascular treatment of wide-neck bifurcation aneurysms. AJNR Am J Neuroradiol 2013;34:1209-14

5. Lubicz B, Klisch J, Gauvrit JY, et al. WEB-DL endovascular treatment of wide-neck bifurcation aneurysms: short- and midterm results in a European study. AJNR Am J Neuroradiol 2014;35:432-38

6. Brinjikji W, Cloft HJ, Kallmes DF. Difficult aneurysms for endovascular treatment: overwide or undertall? AJNR Am J Neuroradiol 2009;30:1513-17

7. Caroff J, Mihalea C, Neki H, et al. Role of C-arm VasoCT in the use of endovascular WEB flow disruption in intracranial aneurysm treatment. AJNR Am J Neuroradiol 2014;35:1353-57

8. CaroffJ, Mihalea C, Dargento F, et al. Woven EndoBridge(WEB) device for endovascular treatment of ruptured intracranial wide-neck aneurysms: a single-center experience. Neuroradiology 2014;56:755-61

9. Kotowski M, Naggara O, Darsaut TE, et al. Safety and occlusion rates of surgical treatment of unruptured intracranial aneurysms: a systematic review and meta-analysis of the literature from 1990 to 2011. J Neurol Neurosurg Psychiatry 2013;84:42-48 